\title{
Avaliação da função pulmonar em crianças e adolescentes com doenças reumáticas
}

\section{autoimunes}

\author{
Evaluation of pulmonary function in children and adolescents with autoimmune rheumatics \\ diseases \\ Evaluación de la función pulmonar em niños y adolescentes com enfermedades reumáticas \\ autoinmunes
}

Vitoria Vilas Boas da Silva Bomfim ORCID: https://orcid.org/0000-0003-4897-0279

Centro Universitário Jorge Amado, Brasil E-mail: pesquisaclinica9@gmail.com

Ana Carolina Socorro Oliveira ORCID: https://orcid.org/0000-0003-1363-7919 Escola Bahiana de Medicina e Saúde Pública, Brasil E-mail: anaoliveira21.1@gmail.com

Maria Eduarda Lopes de Macedo Bezerra ORCID: https://orcid.org/0000-0001-6741-3190 Faculdade Estácio Rio Grande do Norte, Brasil E-mail: eduardalopes022@gmail.com

Emily da Silva Eberhardt ORCID: https://orcid.org/0000-0003-2736-5686 Centro Universitário Cenecista de Osório, Brasil E-mail: milyeberhardt@gmail.com

Vitória dos Santos Cruz ORCID: https://orcid.org/0000-0002-6773-7424 Faculdade Pitágoras Teixeira de Freitas, Brasil E-mail: vitoria0snt@gmail.com

Maria Dhescyca Ingrid Silva Arruda ORCID: https://orcid.org/0000-0002-9073-7844

Faculdade São Francisco da Paraíba, Brasil E-mail: dhescycaingrid20@gmail.com

Hozana Fernanda da Silva ORCID: https://orcid.org/0000-0002-7210-4859 Universidade Maurício de Nassau, Brasil E-mail: hznfernanda@gmail.com

Ludmilla Rafaela Marinho da Silva ORCID: https://orcid.org/0000-0003-0025-2630 Pontifícia Universidade Católica de Minas Gerais, Brasil

E-mail: ludmillarafaela30@gmail.com

Mirela Figueirôa Vieira de Alencar ORCID: https://orcid.org/0000-0002-4230-143X Universidade Tiradentes, Brasil E-mail: mirellaalencar2015@gmail.com

Priscila de Freitas Ferreira ORCID: https://orcid.org/0000-0002-8716-7801

Universidade Federal de Juiz de Fora, Brasil E-mail: prifferreira84@gmail.com Gustavo Silva Brasil

ORCID: https://orcid.org/0000-0002-6512-6443 Universidade Federal de Campo Grande, Brasil E-mail: gustavosbrasilc@icloud.com

Hernandes Flanklin Carvalho Oliveira ORCID: https://orcid.org/0000-0003-0498-0600 Universidade Estadual do Piauí, Brasil E-mail: franklin.oliveira@yahoo.com

Gabriela Mendonça Monte ORCID: https://orcid.org/0000-0002-0935-4548

Faculdade São Francisco da Paraíba, Brasil E-mail: gmonte703@gmail.com 


\title{
Resumo
}

Objetivo: Avaliar a função pulmonar em pacientes com doenças reumáticas autoimunes juvenis, bem como descrever as principais alterações encontradas nos testes de função pulmonar ao longo das consultas médicas de rotina. Metodologia: Revisão integrativa de literatura realizada nas bibliotecas e bases de dados MEDLINE, PubMed, LILACS), Scielo, Biblioteca Cochrane e BIREME. Publicados no recorte temporal de 10 anos (junho de 2011 a junho de 2021). Não haverá restrições no que diz respeito à linguagem, localidade de condução da pesquisa ou ano de publicação. Serão excluídos artigos duplicados, relatos com menos de 5 casos e editoriais, além dos artigos que não atendam aos critérios de inclusão supracitados. Resultados: Os estudos apontam um alto índice de crianças com comprometimento pulmonar relacionado a doença do tecido conjuntivo. A doença do tecido conjuntivo pode resultar em outras complicações como a hipertensão arterial pulmonar, a detecção precoce por ecocardiografia Doppler transtorácica é fundamental na avaliação da hipertensão arterial pulmonar, sendo um método não invasivo. Considerações Finais: A detecção precoce de alterações pulmonares é fundamental e pode ser realizada através da realização de testes das funções pulmonares e um acompanhamento multidisciplinar contínuo, sendo primordial para minimizar os impactos de progressão e complicações de doenças pulmonares.

Palavras-chave: Testes de função respiratória; Doenças reumáticas; Criança; Adolescente; Doenças autoimunes.

\begin{abstract}
Objective: To evaluate lung function in patients with juvenile autoimmune rheumatic diseases, as well as describe the main changes found in pulmonary function tests during routine medical consultations. Methodology: Integrative literature review performed in MEDLINE, PubMed, LILACS, Scielo, Cochrane Library and BIREME libraries and databases. Published in the 10-year time frame (June 2011 to June 2021). There will be no restrictions regarding the language, location of the research or year of publication. Duplicate articles, reports with less than 5 cases and editorials will be excluded, in addition to articles that do not meet the aforementioned inclusion criteria. Results: Studies show a high rate of children with pulmonary impairment related to connective tissue disease. Connective tissue disease can result in other complications such as pulmonary arterial hypertension, early detection by transthoracic Doppler echocardiography is essential in the assessment of pulmonary arterial hypertension, being a non-invasive method. Final Considerations: Early detection of pulmonary alterations is essential and can be performed through tests of pulmonary functions and continuous multidisciplinary follow-up, being essential to minimize the impacts of progression and complications of pulmonary diseases.
\end{abstract}

Keywords: Respiratory function tests; Rheumatic diseases; Child; Adolescent; Autoimmune diseases.

\section{Resumen}

Objetivo: Evaluar la función pulmonar en pacientes con enfermedades reumáticas autoinmunes juveniles, así como describir los principales cambios encontrados en las pruebas de función pulmonar durante las consultas médicas de rutina. Metodología: Revisión integrativa de la literatura realizada en MEDLINE, PubMed, LILACS, Scielo, Cochrane Library y bibliotecas y bases de datos BIREME. Publicado en el plazo de 10 años (junio de 2011 a junio de 2021). No habrá restricciones con respecto al idioma, la ubicación de la investigación o el año de publicación. Se excluirán artículos duplicados, reportajes con menos de 5 casos y editoriales, además de los artículos que no cumplan con los criterios de inclusión antes mencionados. Resultados: Los estudios muestran una alta tasa de niños con deterioro pulmonar relacionado con la enfermedad del tejido conectivo. La enfermedad del tejido conectivo puede resultar en otras complicaciones como la hipertensión arterial pulmonar, la detección precoz mediante ecocardiografía Doppler transtorácica es fundamental en la valoración de la hipertensión arterial pulmonar, al ser un método no invasivo. Consideraciones finales: La detección precoz de las alteraciones pulmonares es fundamental y se puede realizar mediante pruebas de funciones pulmonares y seguimiento multidisciplinario continuo, siendo fundamental para minimizar los impactos de progresión y complicaciones de las enfermedades pulmonares.

Palabras clave: Pruebas de función respiratoria; Enfermedades reumáticas; Niños; Adolescentes; Enfermedades autoinmunes.

\section{Introdução}

Entre as doenças reumáticas, existem as Doenças do Tecido Conectivo (DTC), caracterizadas por um quadro inflamatório e autoimune e frequentemente apresentam alterações na função pulmonar. As principais doenças do tecido 
conectivo que apresentam manifestações pulmonares são artrite reumatoide, lúpus eritematoso sistêmico, esclerose sistêmica, dermatopolimiosite, síndrome de Sjögren e doença mista do tecido conectivo (Baldi, Costa \& Kairalla, 2016). As manifestações mais frequentemente descritas são as Doenças Intersticiais Pulmonares (DIP), caracterizadas por intensa inflamação e fibrose do parênquima pulmonar, mas pleurite e hipertensão pulmonar também são manifestações descritas e o sistema respiratório pode está envolvido em qualquer um dos seus componentes, como músculos, vasos, parênquima, vias aéreas e pleuras (Peradzyńska, Krenke, Szylling, Kołodziejczyk, Gazda, Rutkowska-Sak \& Kulus, 2016). Tais achados justificam a pesquisa de auto-anticorpos em pacientes com diagnóstico de doença pulmonar intersticial sem uma etiologia definida, com o intuito de confirmar ou excluir uma origem reumatológica (Pereira, Kawassaki \& Baldi, 2013). No entanto, cada doença tem um padrão mais peculiar de envolvimento pulmonar, podendo se manifestar mais cedo ou mais tarde em seu curso de evolução. No Lúpus Eritematoso Sistêmico (LES), por exemplo, o acometimento pulmonar é mais comum nos homens, apesar da maior prevalência do LES em mulheres; se dá mais tardiamente na evolução da doença e, é muito comum o envolvimento pleural (Ha, Lee \& Kang, 2018).

Dos pacientes com DIP, até 20\% apresentam alguma doença do tecido conectivo associada (Costa, Dias \& Kairalla, 2013). O diagnóstico da doença intersticial pulmonar associada á doença do tecido conectivo (DIP-DTC) é muito difícil, principalmente quando a afecção pulmonar é a única manifestação, sem um diagnóstico prévio de doença reumática autoimune e exige a colaboração multidisciplinar de reumatologistas, pneumologistas, radiologistas e patologistas (Pereira et al., 2013; Henry, Little, Veeraraghavan, Bhalla \& Elicker, 2016). Além disso, existem muitas causas de DIP, como a fibrose pulmonar idiopática, a pneumonite de hipersensibilidade, a sarcoidose, a pneumonia intersticial não específica associada ou não a doenças do tecido conjuntivo, as doenças ocupacionais, a pneumonia em organização e as bronquiolites diversas (Baldi, Pereira, Rubin, Santana, Costa, Carvalho \& Pimenta, 2012).

Para o diagnóstico de DIP-DTC podem ser utilizados exames de imagem como radiografia e tomografia computadorizada de alta resolução, a qual é mais sensível que a radiografia. Podem também ser utilizados os lavados broncoalveolares, restritos aos pacientes com sinais e sintomas de envolvimento pulmonar, biópsias de pulmão e testes de função pulmonar (TFP). Estes últimos são mais utilizados pelo seu caráter não invasivo e menor custo. Os exames diagnósticos de DIP-DTC são os mesmos para crianças e adultos, o que pode ocorrer em maiores dificuldades diagnósticas para aquelas, como exames de imagem sem alterações, exposição das crianças a altas doses de radiação e impossibilidade de realização dos TFP’s por falta de colaboração entre operador e paciente (Peradzynska et al., 2016).

Apesar de o pulmão ser um órgão frequentemente acometido nas DTC's manifestações pulmonares não são adotadas como parte dos critérios diagnósticos (Henry et al., 2016). Além disso, existem aqueles pacientes que não se enquadram nos critérios diagnósticos de DTC, excetuando-se a esclerose sistêmica, na qual doença intersticial pulmonar é um dos critérios diagnósticos (Henry et al., 2016). Além disso, existem aqueles pacientes que não se enquadram nos critérios de classificação atualmente existentes, sendo, portanto, classificados como portadores de doença do tecido conectivo indiferenciada (DTCI) (Ferri, Manfredi, Sebastiani, Colaci, Giuggioli, Vacchi \& Luppi, 2016). Fischer et al., propôs uma nova classificação para esses pacientes com DIP e um quadro sugestivo de DTCI: Doença do Tecido Conectivo Pulmão-Dominante (Pereira, Dias, Almeida, Araujo, Kawano-Dourado, Baldi \& Carvalho, 2015).

As alterações na função pulmonar podem não apresentar manifestações clínicas, podendo ser completamente assintomáticas e sem achados patológicos nos exames de imagem, principalmente em crianças (Dell, Cernelc-Kohan \& Hagood, 2012). Contudo, alterações no TFP's já podem ser detectados mesmo antes do desenvolvimento de manifestações clínicas. Alkady, Helmy \& Mohamed-Hussein (2012). estudaram as funções cardíacas e pulmonares em 45 crianças com artrite idiopática juvenil (AIJ) sem sintomas cardíacos ou pulmonares, comparados a 36 crianças do grupo controle e, identificaram uma diminuição da capacidade vital forçada ( $\mathrm{FVC} \%$ ), do volume expiratório forçado no primeiro segundo ( $\left.\mathrm{FEV}_{1} \%\right)$, no pico 
do fluxo expiratório (PEV), nas pressões expiratória e inspiratória máximas (Pemax e Pimax, respectivamente) e na capacidade de difusão do monóxido de carbono pelo pulmão (DLCO) nas crianças doentes em comparação aos controles saudáveis.

Quezada Infante, Ramos, García Mora, Norambuena e Pavon (2012). Sugerem que TFP's devem ser feitos pelo menos uma vez no ano nas crianças com doenças reumáticas para prevenção de agravamentos futuros. Alkady et al., 2012 também sugerem a avaliação de rotina da função pulmonar em crianças com AIJ. Na esclerodermia sistêmica, uma doença reumática autoimune cuja principal causa de mortalidade é o comprometimento pulmonar, o recomendável é que se façam testes de função pulmonar e tomografias computadorizadas de alta resolução frequentes, pelo menos nos primeiros cinco anos do diagnóstico da doença em crianças, pois a função pulmonar tende a se estabilizar após esse período. Embora manifestações pulmonares em crianças com doenças reumáticas autoimunes não sejam muito frequentes, quando aparecem estão relacionadas a casos graves, com alta morbidade e mortalidade (Dell et al., 2012).

As dificuldades em se estabelecer uma correlação entre as alterações pulmonares e as doenças reumáticas autoimunes residem no fato de que essas doenças possuem outros fatores que interferem na função pulmonar, como o tratamento com metotrexato, corticosteroides orais e anti-inflamatórios não esteroidais, além da alta incidência de doenças infecciosas e o comprometimento de outros órgãos como coração e rins (Dell et al., 2012; Pereira et al.,2013). Diante do exposto, este estudo se objetiva em avaliar a função pulmonar em pacientes com doenças reumáticas autoimunes juvenis, bem como descrever as principais alterações encontradas nos testes de função pulmonar ao longo das consultas médicas de rotina.

\section{Metodologia}

Trata-se de uma revisão integrativa da literatura, este tipo de estudo se dá por meio de seis etapas sendo elas elaboração da pergunta norteadora, busca na literatura científica, coleta de dados dos artigos escolhidos, análise crítica e reflexiva dos estudos selecionados, discussão dos resultados e apresentação em forma de revisão integrativa (Souza, Silva \& Carvalho, 2010).

Onde foi utilizado bases e bibliotecas de dados eletrônicos Medical Literature Analysis and Retrieval System Online (MEDLINE), U.S. National Library of Medicine (PubMed), Literatura Latino-americana e do Caribe em Ciências da Saúde (LILACS), Scientific Electronic Library Online (Scielo) e Biblioteca Cochrane, Biblioteca Regional de Medicina (BIREME). As buscas serão feitas com auxílio das palavras chaves e Keywords conforme mostra o Quadro 1. 
Quadro 1 - Método de busca utilizado nas plataformas, a fim de encontrar os artigos para revisão. Salvador, 2021.

\begin{tabular}{|c|c|}
\hline BASE DE DADOS & MÉTODO DE BUSCA \\
\hline $\begin{array}{l}\text { MEDLINE/ } \\
\text { PubMed }\end{array}$ & 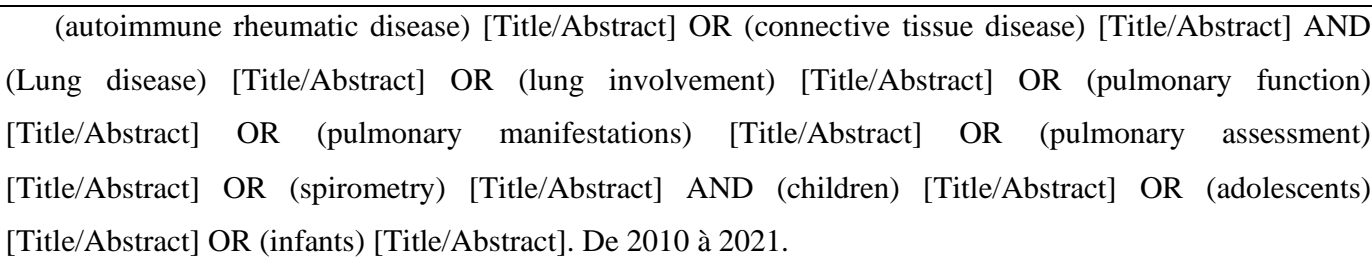 \\
\hline Scielo & $\begin{array}{l}\text { (autoimmune rheumatic disease) [Todos os índices] OR (connective tissue disease) [Todos os índices] } \\
\text { AND (Lung disease) [Todos os índices] OR (lung involvement) [Todos os índices] OR (pulmonary function) } \\
\text { [Todos os índices] OR (pulmonary manifestations) [Todos os índices] OR (pulmonary assessment) [Todos } \\
\text { os índices] OR (spirometry) [Todos os índices] AND (children) [Todos os índices] OR (adolescents) [Todos } \\
\text { os índices] OR (infants) [Todos os índices]. De } 2010 \text { à } 2021 .\end{array}$ \\
\hline LILACS & $\begin{array}{l}\text { (autoimmune rheumatic disease) [Words] OR (connective tissue disease) [Words] AND (Lung disease) } \\
\text { [Words] OR (lung involvement) [Words] OR (pulmonary function) [Words] OR (pulmonary manifestations) } \\
\text { [Words] OR (pulmonary assessment) [Words] OR (spirometry) [Words] AND (children) [Words] OR } \\
\text { (adolescents) [Words] OR (infants) [Words]. De } 2010 \text { à } 2021 .\end{array}$ \\
\hline BIREME & $\begin{array}{l}\text { (autoimmune rheumatic disease) [Search all text] OR (connective tissue disease) [Search all text] AND } \\
\text { (Lung disease) [Search all text] OR (lung involvement) [Search all text] OR (pulmonary function) [Search } \\
\text { all text] OR (pulmonary manifestations) [Search all text] OR (pulmonary assessment) [Search all text] OR } \\
\text { (spirometry) [Search all text] AND (children) [Search all text] OR (adolescents) [Search all text] OR } \\
\text { (infants) [Search all text]. De } 2010 \text { à 2021. }\end{array}$ \\
\hline
\end{tabular}

Fonte: Autores (2021).

Serão incluídos no estudo artigos originais observacionais (estudos de coorte, estudos retrospectivos e estudo de caso) que abordem o tema "doenças reumáticas autoimunes e função pulmonar na população de 0-18 anos" publicados no recorte temporal de 10 anos (junho de 2011 a junho de 2021). Não haverá restrições no que diz respeito à linguagem, localidade de condução da pesquisa ou ano de publicação. Serão excluídos artigos duplicados, relatos com menos de 5 casos e editoriais, além dos artigos que não atendam aos critérios de inclusão supracitados.

A seleção dos estudos encontrados nas bases de dados foi realizada de maneira independente por três revisores (2 pesquisadoras e a orientadora científica) através da avaliação de título, resumo, leitura do artigo integral quando identificados como potencialmente elegíveis. Discordâncias serão ajustadas após discussão. As referências bibliográficas dos artigos selecionados pela leitura do título e resumo também serão avaliadas a fim de se incluir artigos que não tenham sido contemplados nas buscas.

Após a seleção dos artigos para revisão, os mesmos serão avaliados através da leitura crítica e as informações dos artigos serão categorizadas em uma matriz de aferição dos dados, a saber autoria, ano, origem, revista onde foi publicado e impacto, desenho do estudo, número de pacientes, diagnóstico da doença reumática juvenil, idade dos pacientes, teste de função pulmonar e resultado, alterações encontradas em exames de imagem, manifestações clínicas pulmonares encontradas, principais conclusões. O produto final dessa revisão será a análise descritiva dos dados coletados. Todos os artigos incluídos na revisão foram aprovados pelos respectivos comitês de ética dos locais de coleta de dados. Para este presente estudo, em acordo com a Resolução CNS-MS, número 196 de 1996, não é necessária a análise pelo Comitê de Ética em Pesquisa (CEP). 


\section{Resultados e Discussões}

Foram encontrados na busca de literatura 3000 artigos, nos quais após a aplicação dos critérios de inclusão e exclusão restaram 29 artigos para a revisão em pares. Após a revisão em pares ficaram 17 artigos a serem lidos na íntegra conforme demonstra a Quadro 2.

Quadro 2 - Dados avaliados nos estudos selecionados. Salvador, 2021.

\begin{tabular}{|c|c|c|c|c|}
\hline ESTUDO & AUTOR E ANO & PERIÓDICO & $\begin{array}{c}\mathrm{N}^{\circ} \text { DE } \\
\text { PARTICIPANTES }\end{array}$ & DESFECHO \\
\hline 1 & $\begin{array}{l}\text { Witczak, } \\
\text { Hetlevik, Sanner, } \\
\text { Barth, Schwartz, } \\
\text { Flat } \varnothing, \& \text { Sjaastad } \\
\text { (2019) }\end{array}$ & $\begin{array}{l}\text { The Journal of } \\
\text { Rheumatology }\end{array}$ & 52 & $\begin{array}{l}\text { O estudo revela através de testes de espirometria } \\
\text { e tomografia computadorizada que a função } \\
\text { pulmonar de pacientes com doença do tecido } \\
\text { conjuntivo juvenil apresentavam-se prejudicada. }\end{array}$ \\
\hline 2 & $\begin{array}{l}\text { Pereira et al. } \\
\quad(2015)\end{array}$ & $\begin{array}{l}\text { Jornal Brasileiro } \\
\text { de Epidemiologia }\end{array}$ & 1998 & $\begin{array}{l}\text { A amostra relata que os pacientes com doença } \\
\text { pulmonar intersticial, apresentaram } \\
\text { anormalidades espirométricas. As características } \\
\text { extratorácicas mais prevalentes foram artralgia, } \\
\text { doença do refluxo gastroesofágico e fenômeno de } \\
\text { Raynaud. }\end{array}$ \\
\hline 3 & $\begin{array}{l}\text { Peradzynska et al. } \\
\qquad(2016)\end{array}$ & $\begin{array}{l}\text { Advances in } \\
\text { Respiratory } \\
\text { Medicine }\end{array}$ & 98 & $\begin{array}{l}\text { A doença do tecido conjuntivo pode acometer } \\
\text { vários órgãos e tecidos, podendo o sistema } \\
\text { respiratório ser acometido. Os procedimentos do } \\
\text { estudo incluíram história médica, exame físico, } \\
\text { radiografia de tórax e TFP (espirometria e } \\
\text { pletismografia de corpo inteiro). A limitação do } \\
\text { fluxo de ar pode ser caracterizada como uma } \\
\text { anormalidade inicial. }\end{array}$ \\
\hline 4 & $\begin{array}{l}\text { Namendys-Silva, } \\
\text { Ocotlán } \\
\text { González-Herrera, } \\
\text { Hernández- } \\
\text { Oropeza, Rivero- } \\
\text { Sigarroa \& } \\
\text { Domínguez- } \\
\text { Cherit (2013) }\end{array}$ & $\begin{array}{c}\text { Revista Médica de } \\
\text { Chile }\end{array}$ & 35 & $\begin{array}{l}\text { O estudo avalia que a hipertensão arterial } \\
\text { pulmonar é uma das complicações causadas pela } \\
\text { doença do tecido conjuntivo, quando agravadas } \\
\text { apresentam ventrículo direito mais comumente } \\
\text { dilatado, hipertrófico e hipocinético. }\end{array}$ \\
\hline 5 & $\begin{array}{l}\text { Pazini, Pietta-Dias } \\
\text { \& Roncada (2020) }\end{array}$ & $\begin{array}{l}\text { Rev. Paul. } \\
\text { Pediatr. }\end{array}$ & 290 & $\begin{array}{l}\text { O estudo busca avaliar a relação de } \\
\text { comprometimento da função pulmonar entre } \\
\text { crianças asmáticas e níveis de atividades físicas } \\
\text { realizados, no entanto, o estudo abordou que não } \\
\text { há relação entre o supracitado nesta população. }\end{array}$ \\
\hline 6 & $\begin{array}{l}\text { Vieira, Alvim, } \\
\text { Carneiro \& } \\
\text { Ibiapina }(2016)\end{array}$ & $\begin{array}{c}\text { J. bras. } \\
\text { peneumol }\end{array}$ & 70 & $\begin{array}{l}\text { A doença falciforme pode comprometer a função } \\
\text { pulmonar, dependendo do comprometimento e da } \\
\text { gravidade da doença falciforme. Neste estudo } \\
\text { foram feitos testes de espirometria e caminhada } \\
\text { de seis minutos, o que sugeriu um olhar atento à } \\
\text { função pulmonar desde a infância. }\end{array}$ \\
\hline
\end{tabular}




\begin{tabular}{|c|c|c|c|c|}
\hline 7 & $\begin{array}{l}\text { Ríos, Sobrero, } \\
\text { Chiesa, Pinchak, } \\
\text { Blasina, } \\
\text { Keshishian \& } \\
\text { Frachelle (2019) }\end{array}$ & $\begin{array}{l}\text { Arch. Pediatr. } \\
\text { Urug. }\end{array}$ & 04 & $\begin{array}{l}\text { A hemorragia alveolar mediada não } \\
\text { imunologicamente é uma patologia rara que afeta } \\
\text { crianças, problemas de coagulação e alterações } \\
\text { hemodinâmicas podem favorecer o } \\
\text { desenvolvimento dessa patologia. }\end{array}$ \\
\hline 8 & $\begin{array}{l}\text { Bravo, Cardemil, } \\
\text { Torrejón, Bozzo, } \\
\text { Boza, Contreras } \\
\text { \& Nuta (2018). }\end{array}$ & Nutr. Hosp & 37 & $\begin{array}{l}\text { Apesar de a maioria dos participantes com fibrose } \\
\text { cística apresentarem função pulmonar normal, o } \\
\text { estudo evidenciou a necessidade de melhorar a } \\
\text { composição corporal a fim de evitar } \\
\text { complicações futuras da função pulmonar. }\end{array}$ \\
\hline 9 & $\begin{array}{l}\text { Ramos, Santana, } \\
\text { Almeida, } \\
\text { Machado Júnior, } \\
\text { Araújo-Filho \& } \\
\text { Salles (2013) }\end{array}$ & $\begin{array}{l}\text { J. bras. } \\
\text { pneumol }\end{array}$ & 67 & $\begin{array}{l}\text { Variáveis espirométricas e da macroestrutura do } \\
\text { sono, assim como o índice de apneia e hipopneia } \\
\text { foram avaliadas e estão relacionados com a } \\
\text { hipoxemia noturna e consequentemente com a } \\
\text { função pulmonar prejudicada. }\end{array}$ \\
\hline 10 & $\begin{array}{l}\text { Donoso, } \\
\text { Hedenström, } \\
\text { Malinovschi \& } \\
\text { Lilja (2020) }\end{array}$ & $\begin{array}{c}\text { Pediatr } \\
\text { Pulmonol }\end{array}$ & 47 & $\begin{array}{l}\text { A atresia esofágica pode resultar em morbidade } \\
\text { respiratória, o estudo analisa que peso ao nascer, } \\
\text { idade no seguimento e episódios de anestesia } \\
\text { geral foram identificados como fatores de risco } \\
\text { para atresia esofágica. }\end{array}$ \\
\hline 11 & $\begin{array}{l}\text { de Gouveia } \\
\text { Belinelo, Nielsen, } \\
\text { Goddard, Platt, } \\
\text { Sena, Robinson \& } \\
\text { Mattes (2020) }\end{array}$ & $\begin{array}{l}\text { BMC Pulmonary } \\
\text { Medicine }\end{array}$ & 23 & $\begin{array}{l}\text { O estudo correlaciona a asma grave com a função } \\
\text { pulmonar anormal, o qual identificou que } \\
\text { aproximadamente } 80 \% \text { da população do estudo } \\
\text { tem prejuízo nas funções do pulmão decorrentes } \\
\text { da gravidade da asma. }\end{array}$ \\
\hline 12 & $\begin{array}{l}\text { Lundberg, Melén, } \\
\text { Thunqvist, } \\
\text { Norman \& } \\
\text { Hallberg (2020) }\end{array}$ & $\begin{array}{c}\text { Pediatric } \\
\text { Pulmonology }\end{array}$ & 172 & $\begin{array}{l}\text { A oscilimetria por impulso é um método mais } \\
\text { viável do que a espirometria para avaliar a função } \\
\text { pulmonar em crianças pequenas, } \\
\text { independentemente da idade gestacional ao } \\
\text { nascer e pode ser considerado uma alternativa em } \\
\text { crianças que não podem realizar espirometria. }\end{array}$ \\
\hline 13 & $\begin{array}{l}\text { Hildebrandt, } \\
\text { Rahn, Kessler, } \\
\text { Speth, Fischer \& } \\
\text { Ballmann (2021) }\end{array}$ & $\begin{array}{c}\text { Pediatric } \\
\text { Rheumatology }\end{array}$ & 19 & $\begin{array}{l}\text { Nossos achados sugerem que o comprometimento } \\
\text { pulmonar funcional precoce já está presente em } \\
\text { crianças com doenças reumáticas. }\end{array}$ \\
\hline 14 & $\begin{array}{l}\text { Bui, Burgess, } \\
\text { Lowe, Perret, } \\
\text { Lodge, Bui \& } \\
\text { Dharmage (2017) }\end{array}$ & $\begin{array}{l}\text { American Journal } \\
\text { of Respiratory and } \\
\text { Critical Care } \\
\text { Medicine }\end{array}$ & 23 & $\begin{array}{l}\text { Com o tempo, muitas crianças com asma grave } \\
\text { apresentaram melhores desfechos clínicos em } \\
\text { função pulmonar. }\end{array}$ \\
\hline 15 & $\begin{array}{l}\text { Mattiello, Mallol, } \\
\text { Fischer, Mocelin, } \\
\text { Rueda \& Sarria } \\
\text { (2010) }\end{array}$ & J. bras pneumol & 77 & $\begin{array}{l}\text { As crianças com bronquiolite obliterante pós- } \\
\text { infecciosa apresentaram um padrão comum de } \\
\text { comprometimento grave da função pulmonar, } \\
\text { caracterizado por uma obstrução importante das } \\
\text { vias aéreas e um expressivo. }\end{array}$ \\
\hline 16 & $\begin{array}{l}\text { Drago, Ruggieri, } \\
\text { Cuttitta, La } \\
\text { Grutta, Ferrante \& } \\
\text { Cibella (2020) }\end{array}$ & $\begin{array}{l}\text { International } \\
\text { Journal of } \\
\text { Environmental } \\
\text { Research and } \\
\text { Public Health }\end{array}$ & 2493 & $\begin{array}{l}\text { Identificou-se que a exposição ao tabaco e } \\
\text { infecções respiratórias são os principais } \\
\text { determinantes de asma na infância, e também } \\
\text { sensibilização alérgica e comprometimento da } \\
\text { função pulmonar. }\end{array}$ \\
\hline
\end{tabular}




\begin{tabular}{|c|c|c|c|c|}
\hline \multirow{2}{*}{17} & $\begin{array}{c}\text { Melo, Lira, Lima, } \\
\text { Vieira, Dias \& } \\
\text { Andrade (2018) }\end{array}$ & $\begin{array}{c}\text { Jornal Brasileiro } \\
\text { de Pneumologia }\end{array}$ & 56 & $\begin{array}{c}\text { Neste estudo, crianças portadoras de cardiopatia } \\
\text { reumática foram submetidas a espirometria, } \\
\text { manovacuometria e teste de caminhada, } \\
\text { observando tolerância reduzida ao exercício } \\
\text { físico e prejuízo na força expiratória nesta } \\
\text { população. }\end{array}$ \\
\hline
\end{tabular}

Fonte: Autores (2021).

Os estudos 1, 2, 3 e 4 apontam um alto índice de crianças com comprometimento pulmonar relacionado a doença do tecido conjuntivo. $\mathrm{O}$ estudo de número 1 revela o comprometimento pulmonar de aproximadamente $69 \%$ da população estudada, no entanto, nas crianças a progressão é moderada, mas pelo potencial de mortalidade em adultos e alguns casos em crianças, essa problemática necessita de atenção (Witczak et al., 2019). O estudo 2 aborda que a doença pulmonar intersticial pode ser a primeira manifestação da doença do tecido conjuntivo, entretanto, observou-se que os pacientes podem apresentar uma ou mais manifestações da doença do tecido conjuntivo, mas não possuir todos os critérios de diagnósticos da mesma, requerendo um estudo aprofundado para elucidar esse achado. Relatam ainda que as manifestações clínicas são leves nesta amostra populacional, com estabilidade pulmonar identificada (Pereira et al., 2015).

Em contrapartida, o estudo 3 identificou que comparado a uma população saudável, sua amostragem teve 4 vezes mais resultados anormais de espirometria, e comprometimento da função pulmonar. Um achado importante revelou inusitadamente que as anormalidades foram sugestivas de fluxo de ar diminuído ao invés de volumes pulmonares reduzidos (Peradzynska et al., 2016). A doença do tecido conjuntivo pode resultar em outras complicações como a hipertensão arterial pulmonar, a detecção precoce por ecocardiografia Doppler transtorácica é fundamental na avaliação da hipertensão arterial pulmonar, sendo um método não invasivo (Ñamendys-Silva et al., 2013).

Outro achado desta revisão, é a tentativa de correlacionar comprometimento pulmonar e asma em crianças, os estudos de número 5, 11, 14 e 16, abordaram essa temática. No entanto, o estudo de número 5 evidencia que não há anormalidade considerável entre atividade física e prejuízo da função pulmonar entre crianças saudáveis e crianças asmáticas, por exemplo. Entretanto, a obstrução das vias áreas consequentes da doença respiratória crônica, mesmo que leve, aumenta a resistência ao fluxo aéreo podendo resultar em comprometimento da função pulmonar (Pazini et al., 2020).

Complementando o estudo 5, a pesquisa de número 11 identifica um prejuízo na ventilação pulmonar nas crianças portadoras de asma, quando comparadas com crianças sem a enfermidade, o achado se concretiza através de exames de imagem como ressonância magnética de gás hiperpolarizado, estando o comprometimento associado a gravidade da asma (de Gouveia Belinelo et al., 2020). O estudo de número 16 corrobora com a evidência de comprometimento pulmonar relacionado à asma, atestando ainda uma estreita relação entre a sensibilização de doenças respiratórias nos primeiros 2 anos de vida com a exposição à fumaça e o tabaco e ainda a prevalência da asma, resultando em prejuízo da função pulmonar na adolescência (Drago et al., 2020).

Na pesquisa número 14, isso se confirma, além do comprometimento pulmonar prejudicar os indivíduos durante a adolescência, este estudo atesta que os danos podem prorrogar até a vida adulta, podendo em longo prazo acontecer o desenvolvimento de síndrome de sobreposição asma e doença pulmonar obstrutiva crônica, assim intervenções precoces são sugeridas para evitar a progressão contínua dessas doenças que impactam a qualidade de vida dos indivíduos (Bui et al., 2017).

Outras patologias aparecem como colaborativas para comprometimento da função pulmonar, a doença falciforme por exemplo, aparece concomitantemente a problemas pulmonares, um quarto da população amostral teve resultados espirométricos anormais, revelando comprometimento pulmonar, ademais, nesta amostra 57\% dos pacientes tinham também diagnóstico de asma, o que enfatiza a importância de supervisionar a função pulmonar da população asmática. O teste de 
caminhada de 6 minutos, foi utilizado nesta pesquisa, buscando correlacionar a dessaturação como diagnóstico precoce também para hipertensão pulmonar. O estudo identificou ainda que há relação entre anormalidade da função pulmonar e os valores de saturação, podendo indicar doença pulmonar crônica na presença de doença falciforme (Vieira et al., 2016).

Outros estudos apontam a relação de doenças e sinais clínicos com o comprometimento pulmonar, um estudo relata a presença de hipoxemia durante o sono em sua amostragem, caracterizada por eventos de dessaturação que causa impacto e modifica a função pulmonar (Ramos et al., 2013). A hemorragia alveolar difusa que é uma doença que atinge os pulmões, também foi estudada nesta revisão, a doença rara envolve a membrana capilar-alveolar que tem função nas trocas gasosas, sendo assim, seu acometimento reflete diretamente o funcionamento pulmonar (Ríos et al., 2019).

O acometimento da fibrose cística, segundo a literatura é mais comum na população caucasiana, Bravo et al. (2018), tiveram por objetivo em seu estudo, associar a função pulmonar com a densidade óssea, no entanto os resultados não apontaram com fidedignidade algum comprometimento pulmonar diretamente ligado composição corporal óssea. Já com atresia esofágica, foram encontrados sinais claros de relação com comprometimento da função pulmonar, como morbidade respiratória, comprometimento ventilatório obstrutivo, podendo a hiperresponsividade brônquica nesses casos ser comparada com a da asma. O risco para tal, aumenta nas crianças com baixo peso ao nascer e idade avançada no acompanhamento, este estudo evidencia a importância de testes de função pulmonar em crianças com atresia esofágica (Donoso et al., 2020).

Vale ressaltar nesta revisão os impactos da prematuridade no desenvolvimento das funções pulmonares, a prematuridade é fator de risco para diversas enfermidades e complicações clínicas. Se tratando da função pulmonar, nesta população os testes são necessários para o processo de cuidado. Para avaliação das vias aéreas a espirometria é padrão ouro, e por isso, é muito utilizada por seus resultados fidedignos, no entanto, este estudo aborda a sugestão da utilização da oscilometria de impulso como estratégia alternativa, por exigir menos cooperação pode ser utilizada nas populações mais jovens, como subsídio para diagnosticar possíveis prejuízos na função pulmonar e auxiliar as estratégias de tratamento (Lundberg et al., 2020).

Outra enfermidade que altera a função pulmonar é a bronquiolite obiliterante pós infecciosa, derivante de diversas complicações, a obstrução de ar acentuada leva danos pertinentes à saúde dos pulmões. Sugere-se como método de avaliação estratégias espirométricas e pletismográficas associadas, para que auxiliem o embate deste problema (Mattiello et al., 2010). Também a cardiopatia reumática apresenta relação a função pulmonar prejudicada, a causa para tal, no entanto, não foi inteiramente elucidada, uma das principais evidências desse achado foi a intolerância ao exercício utilizando o teste de caminhada de 6 minutos e diminuição da força muscular expiratória, estando esta população em condição de vida desconfortável, e seu bem-estar prejudicado (Melo et al., 2018).

Os sinais de comprometimento pulmonar, como tosse seca, aperto no peito e falta de ar, estão na maioria das vezes ausentes em crianças com doenças reumáticas, no entanto, o comprometimento pode ser existente mesmo na ausência de sintomas, atestado através de pesquisas um prejuízo acentuado das funções das vias aéreas, sendo critério diagnóstico e controle de danos uma avaliação interdisciplinar criteriosa desde a infância (Hildebrandt et al., 2021).

\section{Considerações Finais}

Diante do exposto, podemos considerar que embora o comprometimento pulmonar relacionado a doenças reumáticas possa ser baixo de acordo com algumas literaturas, se relacionar aos sinais e sintomas clínicos, pode ser existente mesmo sem manifestações clínicas prévias. A detecção precoce de alterações pulmonares é fundamental e pode ser realizada através da realização de testes das funções pulmonares e um acompanhamento multidisciplinar contínuo, sendo primordial para minimizar os impactos de progressão e complicações de doenças pulmonares. Faz-se necessários mais estudos acerca da 
temática a fim de respaudar as práticas clínicas e intervenções preconizadas, reduzindo custos e eventos adversos relacionados, obtendo assim melhora clínica mais efetiva para os pacientes.

\section{Referências}

Alkady, E. A., Helmy, H. A., \& Mohamed-Hussein, A. A. (2012). Assessment of cardiac and pulmonary function in children with juvenile idiopathic arthritis. Rheumatology international, 32(1), 39-46.

Baldi, B. G., Costa, A. N., \& Kairalla, R. A. (2016). Manisfestações Pulmonares da Doenças do tecido Conectivo. In Clínica Médica. Manole.

Baldi, B. G., Pereira, C. A. D. C., Rubin, A. S., Santana, A. N. D. C., Costa, A. N., Carvalho, C. R. R., \& Pimenta, S. P. (2012). Destaques das diretrizes de doenças pulmonares intersticiais da Sociedade Brasileira de Pneumologia e Tisiologia. Jornal Brasileiro de Pneumologia, 38(3), $282-291$.

Bravo, P., Cardemil, P. B., Torrejón, C., Bozzo, R., Boza, M. L., Contreras, I., \& Nuta, G. W. (2018). Bone mineral density, lung function, vitamin D and body composition in children and adolescents with cystic fibrosis: a multicenter study. Nutrición hospitalaria: Organo oficial de la Sociedad española de nutrición parenteral y enteral, 35(4), 789-795.

Bui, D. S., Burgess, J. A., Lowe, A. J., Perret, J. L., Lodge, C. J., Bui, M., \& Dharmage, S. C. (2017). Childhood lung function predicts adult chronic obstructive pulmonary disease and asthma-chronic obstructive pulmonary disease overlap syndrome. American journal of respiratory and critical care medicine, 196(1), 39-46.

Costa, A. N., Dias, O. M., \& Kairalla, R. A. (2013). Doenças Intersticiais Pulmonares Associadas às Doenças do Tecido Conjuntivo. Pulmão RJ, 22(1), 26-32.

de Gouveia Belinelo, P., Nielsen, A., Goddard, B., Platt, L., Sena, C. R. D. S., Robinson, P. D., \& Mattes, J. (2020). Clinical and lung function outcomes in a cohort of children with severe asthma. BMC pulmonary medicine, 20(1), 1-9.

Dell, S., Cernelc-Kohan, M., \& Hagood, J. S. (2012). Diffuse and interstitial lung disease and childhood rheumatologic disorders. Current opinion in rheumatology, 24(5), 530-540.

Donoso, F., Hedenström, H., Malinovschi, A., \& E. Lilja, H. (2020). Pulmonary function in children and adolescents after esophageal atresia repair. Pediatric pulmonology, 55(1), 206-213.

Drago, G., Ruggieri, S., Cuttitta, G., La Grutta, S., Ferrante, G., \& Cibella, F. (2020). Determinants of Allergic Sensitization, Asthma and Lung Function: Results from a Cross-Sectional Study in Italian Schoolchildren. International journal of environmental research and public health, $17(14), 5087$.

Ferri, C., Manfredi, A., Sebastiani, M., Colaci, M., Giuggioli, D., Vacchi, C., \& Luppi, F. (2016). Interstitial pneumonia with autoimmune features and undifferentiated connective tissue disease: our interdisciplinary rheumatology-pneumology experience, and review of the literature. Autoimmunity reviews, 15(1), 61-70.

Ha, Y. J., Lee, Y. J., \& Kang, E. H. (2018). Lung involvements in rheumatic diseases: update on the epidemiology, pathogenesis, clinical features, and treatment. BioMed research international, 2018.

Henry, T. S., Little, B. P., Veeraraghavan, S., Bhalla, S., \& Elicker, B. M. (2016). The spectrum of interstitial lung disease in connective tissue disease. Journal of thoracic imaging, 31(2), 65-77.

Hildebrandt, J., Rahn, A., Kessler, A., Speth, F., Fischer, D. C., \& Ballmann, M. (2021). Lung clearance index and diffusion capacity for CO to detect early functional pulmonary impairment in children with rheumatic diseases. Pediatric Rheumatology, 19(1), 1-5.

Lundberg, B., Melén, E., Thunqvist, P., Norman, M., \& Hallberg, J. (2020). Agreement between spirometry and impulse oscillometry for lung function assessment in 6-year-old children born extremely preterm and at term. Pediatric Pulmonology, 55(10), $2745-2753$.

Mattiello, R., Mallol, J., Fischer, G. B., Mocelin, H. T., Rueda, B., \& Sarria, E. E. (2010). Pulmonary function in children and adolescents with postinfectious bronchiolitis obliterans.

Melo, A. L. S. D., Lira, Y. F. B. D., Lima, L. A. B., Vieira, F. C., Dias, A. S., \& Andrade, L. B. D. (2018). Tolerância ao exercício, função pulmonar, força muscular respiratória e qualidade de vida em crianças e adolescentes com cardiopatia reumática. Revista Paulista de Pediatria, 36(2), $199-206$.

Ñamendys-Silva, S. A., Ocotlán González-Herrera, M., Hernández-Oropeza, J. L., Rivero-Sigarroa, E., \& Domínguez-Cherit, G. (2013). Hipertensión arterial pulmonar por ecocardiografía en pacientes con enfermedades de tejido conectivo. Revista médica de Chile, 141(1), 58-62.

Pazini, F., Pietta-Dias, C., \& Roncada, C. (2020). Relationship Between Schoolchildren's Levels Of Physical Activity, Anthropometric Indices And Pulmonary Function. Revista Paulista de Pediatria, 39.

Peradzyńska, J., Krenke, K., Szylling, A., Kołodziejczyk, B., Gazda, A., Rutkowska-Sak, L., \& Kulus, M. (2016). Abnormalities in lung volumes and airflow in children with newly diagnosed connective tissue disease. Advances in Respiratory Medicine, 84(1), 22-28.

Pereira, D. A. S., Dias, O. M., Almeida, G. E. D., Araujo, M. S., Kawano-Dourado, L. B., Baldi, B. G., \& Carvalho, C. R. R. (2015). Lung-dominant connective tissue disease among patients with interstitial lung disease: prevalence, functional stability, and common extrathoracic features. Jornal Brasileiro de Pneumologia, 41(2), 151-160.

Pereira, D. A. S., Kawassaki, A. D. M., \& Baldi, B. G. (2013). Interpretação da positividade de autoanticorpos na doença pulmonar intersticial e colagenose pulmão dominante. Jornal Brasileiro de Pneumologia, 39(6), 728-741.

Quezada Infante, R., Ramos, S., García Mora, M., Norambuena, X., \& Pavon, D. (2012). Lung involvement in rheumatologic diseases in children. 
Research, Society and Development, v. 10, n. 8, e11410817036, 2021

(CC BY 4.0) | ISSN 2525-3409 | DOI: http://dx.doi.org/10.33448/rsd-v10i8.17036

Ramos, R. T. T., Santana, M. A. P., Almeida, P. D. C., Machado Júnior, A. D. S., Araújo-Filho, J. B., \& Salles, C. (2013). Nocturnal hypoxemia in children and adolescents with cystic fibrosis. Jornal Brasileiro de Pneumologia, 39(6), 667-674

Ríos, M., Sobrero, H., Chiesa, P., Pinchak, C., Blasina, F., Keshishian, R., \& Frachelle, M. H. (2019). Hemorragia alveolar difusa no inmune en pediatría. Archivos de Pediatría del Uruguay, 90(2), 83-89.

Souza, M. T. D., Silva, M. D. D., \& Carvalho, R. D. (2010). Revisão integrativa: o que é e como fazer. Einstein (São Paulo), 8(1), 102-106.

Vieira, A. K., Alvim, C. G., Carneiro, M. C. M., \& Ibiapina, C. D. C. (2016). Pulmonary function in children and adolescents with sickle cell disease: have we paid proper attention to this problem? Jornal Brasileiro de Pneumologia, 42(6), 409-415.

Witczak, B. N., Hetlevik, S. O., Sanner, H., Barth, Z., Schwartz, T., Flatø, B., \& Sjaastad, I. (2019). Effect on cardiac function of longstanding juvenile-onset mixed connective tissue disease: A controlled study. The Journal of rheumatology, 46(7), 739-747. 